\title{
Implementation of the modeling and simulation software of search and rescue system based Korea navigation satellite system
}

\author{
Incheol Jeong ${ }^{1}$, Sanguk Lee ${ }^{1}$, Woo-Geun $\mathrm{Ahn}^{2}$ \\ ${ }^{1}$ Satellite Research Department, ETRI \\ ${ }^{2}$ Agency for Defense Development
}

\begin{abstract}
This paper describes the development of M\&S (Modeling and Simulation) system based COSPAS-SARSAT SAR (Search and Rescue) system. SAR system transmits a distress signal to the terminal and transmits it to the satellite network or the terrestrial network. It recognized distress incident from anywhere in the world. The M\&S system is designed to analyze the accuracy, reliability, and availability of the SAR (Search and Rescue) system. It is also designed to simulate the actual distress beacons of a distress situation. It was developed to enable performance analysis. We will verify the validity of the implemented results, applying the RF signal processing based on KNSS.
\end{abstract}

\section{Introuction}

In this paper, we investigate the accuracy, reliability, and availability of the SAR (Search and Rescue) system when constructing the M\&S (Modelling and Simulation) system based on the concept of the existing military SAR and the result of analyzing the civil search structure system based on COSPAS-SARSAT (Cosmicheskaya Sistema Poiska Avariynich Sudov - Search and Rescue SatelliteAided Tracking). SAR system can provide distress alert and location information to SAR service if land and aircraft distress incident occurs from anywhere in the world.

When the search structure terminal is activated, a distress signal of $406 \mathrm{MHz}$ is emitted (1), the structure signal is received from the COSPASSARSAT composed of low-earth orbit satellite (2), relayed by the local receiver station (LUT) (3), the ground receiving station calculates the position of the search structure terminal and transmits it to the Rescue Coordination Centre (RCC) through the MCC (MCC) (5), and the RCC performs the rescue operation do.

The purpose of this paper is to measure the accuracy, reliability, and availability performance of the search structure system and to present the direction of the COSPAS-SARSAT search structure system by building a test bed.

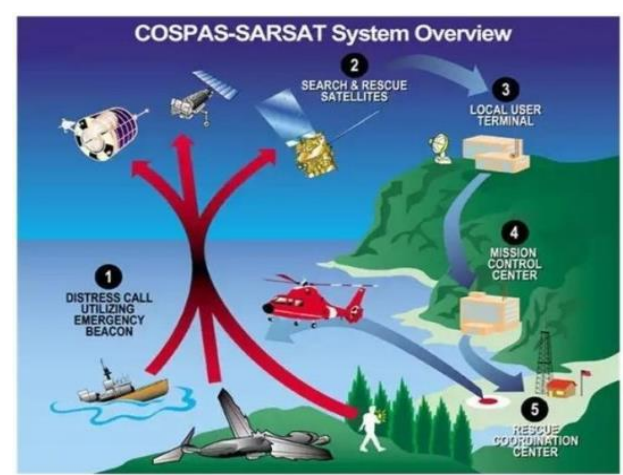

Fig. 1 Flow of the COSPAS-SARSAT SAR

We describe the results of analyzing the accuracy, reliability, and availability of the SAR system based on the M\&S system of the RF signalbased military search and rescue system through COSPAS-SARSAT and applying the dynamics model to the USRP test bed.

In this paper, functional scope of the simulator, the architecture of the SAR M\&S Software will be presented. It is ensured that SAR system satisfies the desired performance in KNSS environment. 


\section{Modelling and simulation of the search and rescue system}

The USRP test bed consists of one transmitter and two receivers, as shown in Fig. 2. The military navigation system is based on the Korea Navigational Satellite System (KNSS), the LTE, and the COSPASSARSAT. . AES 128bit algorithm is implemented and a static signal is implemented by developing the COSPAS-SARSAT (C/S) Spread Spectrum specification and satisfying the second generation COSPAS-SARSAT standard.

The USRP system was used to implement the RF signal-based search structure system, and the accuracy, reliability, and availability performance of the COSPAS- SARSAT search structure system were measured using the on-board dynamics model.

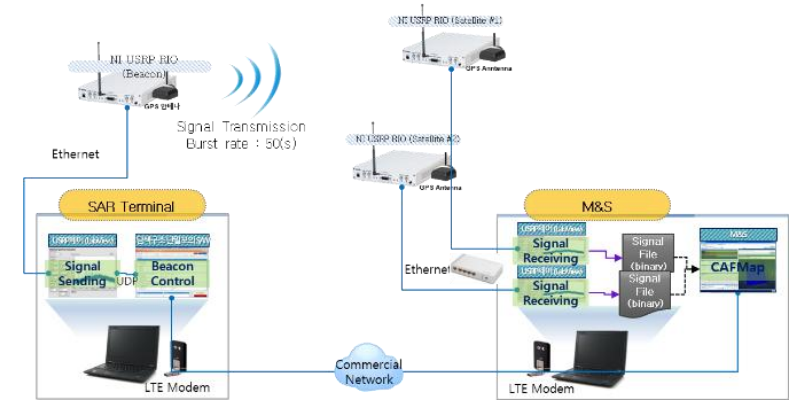

Figure 2 Search and Rescue System based USRP

M\&S software of military navigation system consists of 4 main modelling domains which are user, communication link, ground station (including mission control centre) and system operating environment as shown in Figure 3.

The User model of the SAR system is a function that automatically sends a distress signal when a distress situation occurs and transmits a distress signal in response to a call signal of the ground station. The system operation environment models GIS (Geometric Information System), weather information, antenna information, and satellite information required to calculate propagation attenuation for terrestrial and satellite signals.

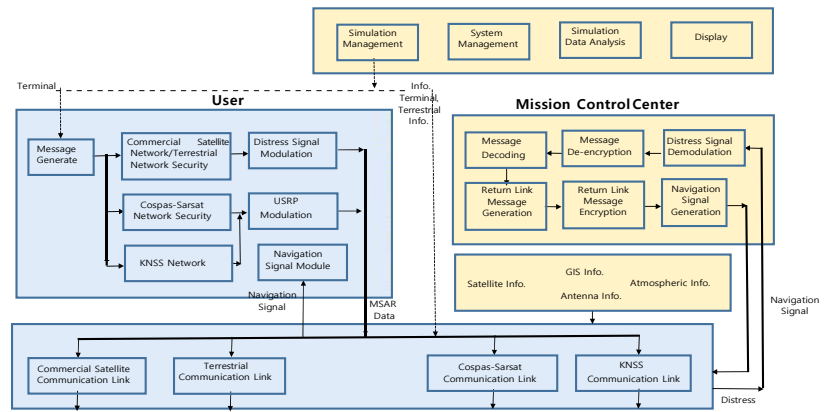

Figure 3 Block Diagram of the Military SAR M\&S Software

In the communication link model, the propagation attenuation of the input transmission signal is composed of the terrestrial network and the satellite network, and the attenuation variable of the operating environment model is reflected on the distance between the terminal and the ground station. The ground station consists of a mission control center, a search and rescue team, and a ground receiving station for ground communication.

The M\&S software of the military SAR system includes satellite orbital generation, availability analysis of beacon terrain and beacon satellites using terrain, analysis of rescue time and beacon operation time in distress, and terrestrial signal and satellite signal reach analysis. It is possible to simulate a military search and rescue structure similar to the actual situation using GIS information.

\section{Search and rescue system and Korea Navigation Satellite System}

COSPAS-SARSAT system was provided by using low-earth orbit satellite and geostationary satellite. There are same types as marine, airborne, and potable handheld navigation devices. They are installed in personal portable devices, ships or aircraft and transmit alarm signal to navigation satellite to inform distress signals.

A military SAR is a system that detects and locates distress signals by mounting them on highspeed vehicles such as satellites. Therefore, rather than loading the equipment into a satellite mobile object, the motion of the high-speed mobile object is simulated by applying a dynamic model. The military SAR system simulates Doppler frequency measurement and actual propagation environment according to satellite motion to reflect the dynamic model.

KNSS system consists of the 3 Geostationary Orbit Satellite (GEO-1, GEO-2, GEO-3) and 4 Elliptical Inclined Geosynchronous Orbit Satellite (EIGSO-1, EIGSO-2, EIGSO-3 and EIGSO-4).

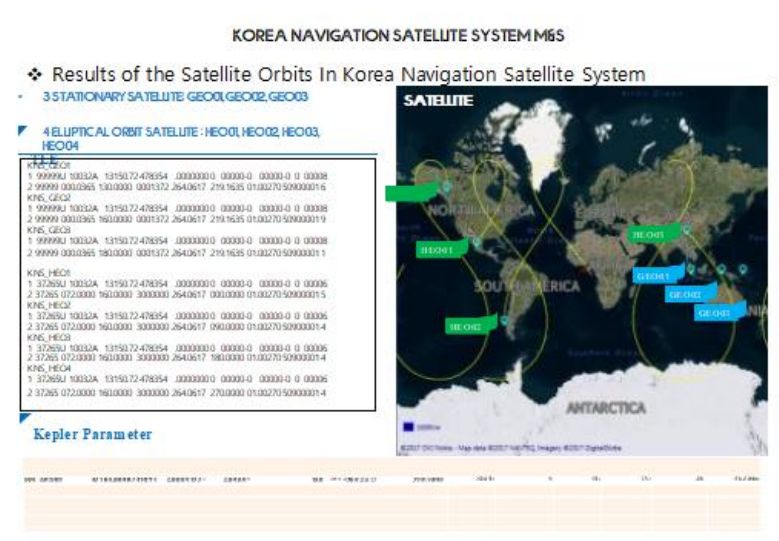

Figure 4 Satellite Orbits in KNSS

As a result of analyzing the satellite orbits of the KNSS, it continuously covers the Korean peninsula and always maintains more than 6 satellites.

Elevation angel of the GEO (Geostationary Orbit) satellites are fixed at $21 \sim 47$ degrees, and 
EIGSO (Elliptical Inclined Geosynchronous Orbit) satellites are $6 \sim 83$ degrees depending on orbit change. The $\mathrm{C} / \mathrm{No}$ for the satellite received signal strength is $36.86 \sim 37.36 \mathrm{~dB}$ for GEO satellites and $34.8 \sim 38.3 \mathrm{~dB}$ for EIGSO satellites.

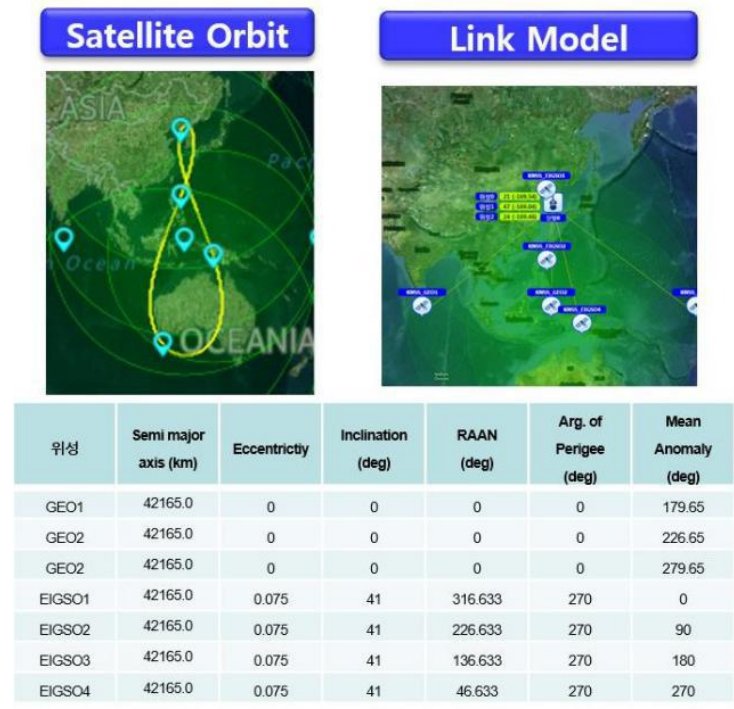

Figure 5 Satellite Orbits in Korea Navigation Satellite System

\section{Analysis of the M\&S of the KNSS}

In KNSS, the EIGSO has a large variation in position accuracy depending on the point of time since the satellite velocity is not constant, and it is difficult to form a geometry due to a lack of number of the satellites.

Therefore, as a result of the performance evaluation simulation, the peak due to the accumulation of CAF (Cross Ambiguity Function) was not formed well. The results were measured to be $1 \mathrm{~km}$ level with ground positioning performance only by elliptical satellite orbit. We think about that it must use LEO (Low Earth Orbit) and MEO (Middle Earth Orbit) satellite same as Galileo and COSPASSARSAT for good geometry.

The performance of the ground positioning in a SAR system was measured to be less than $1 \mathrm{KM}$ only by the combination of the KNSS. This means that the location of the distress incident has an error of about $1 \mathrm{KM}$ compared to the location identified by the system. After that, ETRI will make efforts to reduce errors by developing algorithms to improve ground positioning performance.

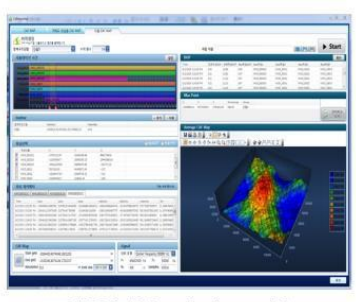

<EIGSO 416 times signal accumulation

Figure 6 Performance Positioning using KNSS

\section{Conclusion}

This paper presents validity of the implemented results about $\mathrm{M} \& \mathrm{~S}$, applying the RF signal processing based on KNSS and applying the dynamics model to the USRP test bed.

At the sending the distress signal, distress terminal generates and transmits a spread spectrum RF signal. At the receiving end, the possibility of the location estimation of the victim was verified based on the signal. In the next approach, ETRI will verify and extend algorithm applied to the module about ground positioning.

\section{Reference}

1. Jeong, I. C., Kim, D. W., Ahn, W. G., \& Lee, S. U., (2017) Development of Search and Rescue System with Dynamic Model by RF Signal Based LTE, Journal of Satellite, information and Communications, Vol 12 No.4, 120-124

2. Kim, J. H., Lee, S. U., Kim, J. H., Ahn, W. G. (2014) Development Status of Military Search and Rescue System M\&S Software, The Journal of Korea Society of Communication and Space Technology, Vol 9 No 3, 121-126 